\title{
FINITE $p$-GROUPS IN WHICH THE NORMAL CLOSURE OF EACH NON-NORMAL CYCLIC SUBGROUP IS NONABELIAN
}

\author{
ZVONIMIR JANKO \\ University of Heidelberg, Germany
}

\begin{abstract}
We determine up to isomorphism finite non-Dedekindian $p$-groups $G$ (i.e., $p$-groups which possess non-normal subgroups) such that the normal closure of each non-normal cyclic subgroup in $G$ is nonabelian. It turns out that we must have $p=2$ and $G$ has an abelian maximal subgroup $A$ of exponent $2^{e}, e \geq 3$, and an element $v \in G-A$ such that for all $h \in A$ we have either $h^{v}=h^{-1}$ or $h^{v}=h^{-1+2^{e-1}}$.
\end{abstract}

Let $G$ be a finite $p$-group. Then it is well known (see [3, Theorem 224.1]) that the normal closure of each cyclic subgroup in $G$ is abelian if and only if each two-generator subgroup of $G$ is of class $\leq 2$. If each two-generator subgroup of a $p$-group $G$ is of class $\leq 2$, then either $G$ is of class $\leq 2$ or $p=3$ and $G$ is of class 3 .

It is natural to ask what happens if $G$ is a non-Dedekindian finite $p$-group in which the normal closure of each non-normal cyclic subgroup is nonabelian. It turns out as a big surprise that in this case we must have $p=2$ and $G$ can be determined up to isomorphism (Theorem 2).

All groups considered here are finite $p$-groups and our notation is standard (see [1]).

DeFINITION 1. Let $M$ be a 2-group possessing an abelian maximal subgroup $H$ of exponent $\geq 4$ such that there is an element $v \in M-H$ which inverts each element of $\bar{H}$. Then $o(v) \leq 4$ since $v$ inverts $\left\langle v^{2}\right\rangle \leq H$.

If $o(v)=2$, then all elements in $M-H$ are involutions and $M$ is called "quasidihedral" (or generalized dihedral).

2010 Mathematics Subject Classification. 20 D15.

Key words and phrases. Finite $p$-groups, normal closure, quasidihedral 2-groups, quasigeneralized quaternion groups, exponent of a $p$-group. 
If $o(v)=4$, then all elements in $M-H$ are of order 4 with the same square $v^{2}$ and then $M$ is called "quasi-generalized quaternion".

TheOREM 2. Let $G$ be a non-Dedekindian p-group in which the normal closure of any non-normal cyclic subgroup is nonabelian. Then $p=2, G$ has an abelian maximal subgroup $A$ of exponent $2^{e}, e \geq 3$, and for an element $v \in G-A$ and for all $h \in A$ we have either $h^{v}=h^{-1}$ or $h^{v}=h^{-1+2^{e-1}}$.

Conversely, let $G$ be a 2-group just defined. Then each subgroup of $A$ is $G$-invariant and for each $v \in G-A, o(v) \leq 4,\langle v\rangle$ is non-normal in $G$ and $\langle v\rangle^{G}=[A,\langle v\rangle]\langle v\rangle$, where $v$ inverts each element of $[A,\langle v\rangle]=G^{\prime}$ (of exponent $2^{e-1} \geq 4$ ) so that $\langle v\rangle^{G}$ is either quasidihedral (in case $o(v)=2$ ) or quasi-generalized quaternion (in case $o(v)=4$ ) and so in any case $\langle v\rangle^{G}$ is nonabelian.

In the proof of Theorem 2, we shall use [2, Theorem 125.1] and therefore we state here that theorem for convenience:

LEMMA 3. Let $G$ be a nonabelian p-group containing a maximal subgroup $H$ such that all subgroups of $H$ are $G$-invariant. Then there is an element $g \in G-H$ such that one of the following holds:

(i) $p=2, H$ is Hamiltonian, i.e., $H=Q \times V$, where $Q \cong \mathrm{Q}_{8}, \exp (V) \leq 2$, and $g \in \mathrm{Z}(G), \mathrm{o}(g) \leq 4$

(ii) $p=2, H$ is abelian of exponent $2^{e}, e \geq 2$, and $g$ either inverts each element in $H$, or $e \geq 3$ and $h^{g}=h^{-1+2^{e-1}}$ for all $h \in H$. In both cases $\mathrm{Z}(G)=\mathrm{C}_{H}(g)=\Omega_{1}(H)$ is elementary abelian and $\mathrm{o}(g) \leq 4$.

(iii) $p=2, H$ is abelian of exponent $2^{e}, e \geq 3$, and $h^{g}=h^{1+2^{e-1}}$ for all $h \in H$, where $\mathrm{Z}(G)=\mathrm{C}_{H}(g)=\Omega_{e-1}(H)$.

(iv) $p>2, H$ is abelian of exponent $p^{e}, e \geq 2$, and $h^{g}=h^{1+p^{e-1}}$ for all $h \in H$, where $\mathrm{Z}(G)=\mathrm{C}_{H}(g)=\Omega_{e-1}(H)$.

Proof of Theorem 2. Suppose that $G$ is a title $p$-group. Let $A<G$ be a maximal normal abelian subgroup of $G$. Since each cyclic subgroup in $A$ is normal in $G$, it follows that each subgroup in $A$ is $G$-invariant.

Suppose $p>2$. Let $B / A$ be a normal subgroup of order $p$ in $G / A$. Applying Lemma 3 on the subgroup $B$, we get $\exp (A)=p^{e}, e \geq 2$, and there is $g \in B-A$ such that for all $h \in A, h^{g}=h^{1+p^{e-1}}$. Since $B$ is nonabelian, there is $b \in B-A$ such that $\langle b\rangle$ is not normal in $B$ (and so $\langle b\rangle$ is not normal in $G)$ so that (replacing $b$ with a suitable power $b^{i}, i \neq \equiv 0(\bmod p)$, if necessary) we have for all $h \in H, h^{b}=h^{1+p^{e-1}}$ and $M=\langle b\rangle^{G}$ is nonabelian with $M \leq B$. Also, $B^{\prime}=[A,\langle b\rangle]$ is elementary abelian and $B^{\prime} \leq \mathrm{Z}(G)$ because each subgroup of $A$ is $G$-invariant. There is $k \in G$ such that $\left[b, b^{k}\right] \neq 1$, where $b^{k} \in M-A$. Note that $\left\langle b^{p}\right\rangle=\langle b\rangle \cap A$ and so $\left\langle b^{p}\right\rangle \unlhd G$ and therefore $\left\langle b^{k}\right\rangle \cap\langle b\rangle=\left\langle b^{p}\right\rangle$. There is $b^{\prime} \in\left\langle b^{k}\right\rangle-A$ such that $\left(b^{\prime}\right)^{p}=b^{-p}$ and $\left[b, b^{\prime}\right] \neq 1$. 
We compute

$$
\left(b b^{\prime}\right)^{p}=b^{p}\left(b^{\prime}\right)^{p}\left[b^{\prime}, b\right]^{\left(\begin{array}{c}
p \\
2
\end{array}\right)}=1,
$$

and so $o\left(b b^{\prime}\right)=p$. Set $b b^{\prime}=s$ and assume $s \in \mathrm{Z}(G)$. But then

$$
\left[b, b^{\prime}\right]=\left[b, b^{-1} s\right]=1,
$$

a contradiction. Thus, $s$ is an element of order $p$ in $B-A$ and $\langle s\rangle$ is not normal in $G$. By our basic assumption, $\langle s\rangle^{G} \leq B$ is nonabelian and so there is $l \in G$ such that setting $s^{\prime}=s^{l} \in B-A$, we have $\left[s, s^{\prime}\right] \neq 1$. But $\left[s, s^{\prime}\right]=z$ is an element of order $p$ in $\mathrm{Z}(G)$ (noting that $B^{\prime} \leq \mathrm{Z}(G)$ and $B^{\prime}$ is elementary abelian). It follows that $K=\left\langle s, s^{\prime}\right\rangle \cong \mathrm{S}\left(p^{3}\right)$ (the nonabelian group of order $p^{3}$ and exponent $\left.p\right)$. Since $K \leq B$, we have $K \cap A \cong \mathrm{E}_{p^{2}}$. On the other hand, $K \cap A \leq \mathrm{Z}(G)$ and $|K:(K \cap A)|=p$ so that $K$ is abelian, a contradiction. We have proved that we must have $p=2$.

Since each subgroup of $A$ is $G$-invariant and $\mathrm{C}_{G}(A)=A$, we have $\exp (A) \geq 4$. Now assume, by way of contradiction, that $\exp (A)=4$. Let $B / A$ be any subgroup of order 2 in $G / A$. By Lemma 3 , each element $b \in B-A$ inverts each element in $A$. This implies that $G / A$ has only one subgroup of order 2 and so $G / A$ is either cyclic or generalized quaternion. Suppose $|G / A|>2$. Then there is $g \in G-A$ such that $(A\langle g\rangle) / A \cong \mathrm{C}_{4}$, where $g^{2}$ inverts each element in $A$. Let $y \in A$ be an element of order 4. Since $\langle y\rangle \unlhd G$, $g$ normalizes $\langle y\rangle \cong \mathrm{C}_{4}$ and $g^{2}$ inverts $\langle y\rangle$, a contradiction. Hence $|G / A|=2$ and so $\exp (G)=4$. Indeed, since each element $v \in G-A$ inverts each element in $A$, we get $o(v) \leq 4$. Because (by our assumption) $G$ is not Dedekindian, there is $v \in G-A$ such that $\langle v\rangle$ is not normal in $G$, where $o(v) \leq 4$. We have $[A,\langle v\rangle]=G^{\prime}$ is elementary abelian and $G^{\prime} \leq \mathrm{Z}(G)$. Set $R=\langle v\rangle^{\bar{G}}$ so that our basic assumption implies that $R$ is nonabelian. On the other hand,

$$
[A,\langle v\rangle]\langle v\rangle \leq R \text { and }[A,\langle v\rangle]\langle v\rangle=G^{\prime}\langle v\rangle \unlhd G
$$

so that $R \leq[A,\langle v\rangle]\langle v\rangle$ and $R=\langle v\rangle^{G}=[A,\langle v\rangle]\langle v\rangle=G^{\prime}\langle v\rangle$.

But $G^{\prime} \leq \mathrm{Z}(G)$ implies that $v$ centralizes $G^{\prime}$ and so $R$ is abelian, a contradiction. We have proved that $\exp (A)=2^{e}$ with $e \geq 3$.

Suppose, by way of contradiction, that $G / A$ possesses a subgroup $B / A$ of order 2 such that for all $b \in B-A$ and $h \in A$, we have $h^{b}=h^{1+2^{e-1}}$. In this case $B^{\prime}=[A,\langle b\rangle]$ is elementary abelian and $B^{\prime} \leq \mathrm{Z}(G)$. Also, we have $\mathrm{Z}(B)=\mathrm{C}_{A}(b)=\Omega_{e-1}(A)$. If $B$ is not normal in $G$, then there is $x \in G$ such $b^{x} \in G-B$ for some $b \in G-A$. But then $b b^{x} \in G-A$ and $b b^{x}$ centralizes $A$ ( since $b^{x}$ acts on $A$ the same way as $b$ does), a contradiction. Hence $B \unlhd G$. Because $B$ is nonabelian and $\exp (B) \geq 8$, it follows that $B$ is not Dedekindian. Hence there is $b \in B-A$ such that $\langle b\rangle$ is not normal in $B$. By our basic assumption, $\langle b\rangle^{G} \leq B$ is nonabelian. There is $g \in G$ such that $b^{g} \in B-A$ and $\left[b, b^{g}\right] \neq 1$. On the other hand, $\left\langle b^{2}\right\rangle \unlhd G$ and so $\langle b\rangle \cap\left\langle b^{g}\right\rangle=\left\langle b^{2}\right\rangle$. There is $c \in\left\langle b^{g}\right\rangle-A$ such that $c^{2}=b^{-2}$ and $[b, c] \neq 1$. We 
compute $(b c)^{2}=b^{2} c^{2}[c, b]=[c, b] \neq 1$. Since $[c, b]$ is of order 2 , it follows that $d=b c$ is an element of order 4 and $d \in A$. Hence $d \in \mathrm{Z}(B)$ and so we get

$$
[b, c]=\left[b, b^{-1} d\right]=\left[b, b^{-1}\right][b, d]=1,
$$

a contradiction. We have proved that such a group $B / A$ of order 2 in $G / A$ does not exist. Then using again Lemma 3 , we see that $G / A$ has exactly one subgroup $C / A$ of order 2 such that for all $c \in C-A$ and $h \in A$, we have either $h^{c}=h^{-1}$ or $h^{c}=h^{-1+2^{e-1}}$. In any case $o(c) \leq 4$.

Assume $|G / A|>2$. Then there is $g \in G-A$ such that $g^{2} \in C-A$ so that if $h$ is an element of order 4 in $A$, then $g$ normalizes $\langle h\rangle \unlhd G$ and $g^{2}$ inverts $\langle h\rangle$, a contradiction. We have proved that $|G / A|=2$, all elements in $G-A$ are of order $\leq 4, \exp (A)=2^{e}, e \geq 3$, and for each $v \in G-A$ and $h \in A$, either $h^{v}=h^{-1}$ or $h^{v}=h^{-1+2^{e-1}}$. The structure of our group $G$ is determined.

Conversely, let $G$ be a 2 -group defined above and let $v$ be any element in $G-A$. Then $o(v) \leq 4$ and the way in which $v$ acts on $A$ insures that each subgroup of $A$ is $G$-invariant. Set $R=\langle v\rangle^{G} \unlhd G$ and then $[A,\langle v\rangle]\langle v\rangle \leq$ $R$. On the other hand, $[A,\langle v\rangle]=G^{\prime}$ and therefore $[A,\langle v\rangle]\langle v\rangle \unlhd G$ so that $R \leq[A,\langle v\rangle]\langle v\rangle$. We have proved that $[A,\langle v\rangle]\langle v\rangle=R$. But $\exp ([A,\langle v\rangle])=$ $2^{e-1} \geq 4$ and so $v$ inverts each element of $[A,\langle v\rangle]=G^{\prime}$ so that $\langle v\rangle^{G}$ is either quasidihedral (if $o(v)=2$ ) or quasi-generalized quaternion (if $o(v)=4$ ). Hence in any case $\langle v\rangle^{G}$ is nonabelian. Our theorem is proved.

\section{REFERENCES}

[1] Y. Berkovich, Groups of prime power order, Vol. 1, Walter de Gruyter, Berlin-New York, 2008.

[2] Y. Berkovich and Z. Janko, Groups of prime power order, Vol. 3, Walter de Gruyter, Berlin-New York, 2011.

[3] Y. Berkovich and Z. Janko, Groups of prime power order, Vol. 5, Walter de Gruyter, Berlin-New York, 2014.

Z. Janko

Mathematical Institute, University of Heidelberg

69120 Heidelberg

Germany

E-mail: janko@mathi.uni-heidelberg.de

Received: 20.1.2014. 